\title{
Erratum to: Fatty acids and amino acids of entomopathogenic fungus Conidiobolus coronatus grown on minimal and rich media
}

\author{
Marek Gołębiowski $^{1}$ - Aleksandra Ostachowska ${ }^{1}$ - Monika Paszkiewicz ${ }^{2}$ • \\ Mieczysława I. Boguś ${ }^{3}$ - Emilia Włóka ${ }^{3}$ - Marta Ligęza-Żuber ${ }^{3} \cdot$ Piotr Stepnowski $^{4}$
}

Published online: 26 April 2017

(C) Institute of Chemistry, Slovak Academy of Sciences 2017

\section{Erratum to: Chem. Pap. \\ DOI: $10.1515 /$ chempap-2016-0067}

Unfortunately, there was an error in the acknowledgements in the original publication of the article.

The correct acknowledgements are:

"Acknowledgements The authors would like to express their gratitude to Paulina Rybicka for her assistance. This study was funded by the National Science Center and the
Polish Ministry of Science and Higher Education (Grants number: UMO-2011/01/D/NZ6/03114 and DS 530-8617D-594-15)."

Instead of: "Acknowledgements The authors would like to express their gratitude to Paulina Rybicka for her assistance. This study was funded by the Polish Ministry of Science and Higher Education (Grants number UMO-2011/ 01/D/NZ6/03114 and DS 530-8617-D-594-15)."'

The online version of the original article can be found under doi:10.1515/chempap-2016-0067.

Marek Gołębiowski

marek.golebiowski@ug.edu.pl

1 Laboratory of Analysis of Natural Compounds, Department of Environmental Analytics, Faculty of Chemistry, University of Gdańsk, ul. Wita Stwosza 63, 80952 Gdańsk, Poland

2 Department of Environmental Analytics, Faculty of Chemistry, University of Gdańsk, ul. Wita Stwosza 63, 80952 Gdańsk, Poland

3 Institute of Parasitology, Polish Academy of Sciences, Twarda 51/55, 00818 Warsaw, Poland

4 Laboratory of Chemical Environmental Risks, Department of Environmental Analytics, Faculty of Chemistry, University of Gdańsk, ul. Wita Stwosza 63, 80308 Gdańsk, Poland 